\title{
Adherence to guidelines in idiopathic pulmonary fibrosis: a follow-up national
} survey

\author{
Vincent Cottin ${ }^{1}$, Emmanuel Bergot ${ }^{2}$, Arnaud Bourdin $^{3}$, Jacques Cadranel ${ }^{4}$, \\ Philippe Camus ${ }^{5}$, Bruno Crestani ${ }^{6}$, Jean-Charles Dalphin ${ }^{7}$, Philippe Delaval $^{8}$, \\ Claire Dromer ${ }^{9}$, Dominique Israel-Biet ${ }^{10}$, Romain Kessler ${ }^{11}$, \\ Sylvain Marchand-Adam ${ }^{12}$, Charles Hugo Marquette ${ }^{13}$, Grégoire Prévot ${ }^{14}$, \\ Martine Reynaud-Gaubert ${ }^{15}$, Dominique Valeyre ${ }^{16}$, Benoit Wallaert ${ }^{17}$, \\ Benoit Bouquillon $^{18}$ and Jean-François Cordier ${ }^{1}$
}

Affiliations: ${ }^{1}$ National Reference Centre for Rare Pulmonary Diseases, Louis Pradel Hospital, Claude Bernard Lyon 1 University, Lyon, France. ${ }^{2}$ University Hospital, Caen, France. ${ }^{3}$ University Hospital, Montpellier, France. ${ }^{4}$ Tenon University Hospital, Paris, France. ${ }^{5}$ University Hospital, Dijon, France. ${ }^{6}$ Bichat University Hospital, Paris, France. ${ }^{7}$ University Hospital, Besançon, France. ${ }^{8}$ University Hospital, Rennes, France. ${ }^{9}$ University Hospital, Bordeaux, France. ${ }^{10}$ European Hospital Georges Pompidou, Paris, France. ${ }^{11}$ University Hospital Civil, Strasbourg, France. ${ }^{12}$ University Hospital, Tours, France. ${ }^{13}$ University Hospital, Nice, France. ${ }^{14}$ University Hospital, Toulouse, France. ${ }^{15}$ University Hospital, Marseille, France. ${ }^{16}$ AP-HP, Avicenne University Hospital, Bobigny, France. ${ }^{17}$ University Hospital, Lille, France. ${ }^{18}$ Opened Mind Health, Lille, France.

Correspondence: Vincent Cottin, Hospices Civils de Lyon, Hôpital Louis Pradel, Service de Pneumologie Centre de Référence National des Maladies Pulmonaires Rares, Université Claude Bernard Lyon 1, UMR754, Lyon, France. E-mail: vincent.cottinđachu-lyon.fr

ABSTRACT A new survey coordinated by the French expert centres for rare pulmonary diseases investigated French pulmonologists' diagnostic and therapeutic practice for idiopathic pulmonary fibrosis (IPF) and explored changes since a previous survey in 2011-2012.

From May 16 to August 30, 2014, 524 pulmonologists were contacted. Those following at least one patient with IPF were invited to complete a questionnaire administered by telephone or e-mail.

$166(31.7 \%)$ pulmonologists, 161 (97\%) of whom had participated to the first survey, completed the questionnaire. Of those, $46 \%$ and $52 \%$, respectively, discussed the cases with radiologists and pathologists. Out of 144 pulmonologists practicing outside of expert centres, $80 \%$ indicated referring patients to those centres. The 2013 French practical guidelines for IPF were known by $92 \%$ of pulmonologists involved in IPF, 96\% of whom considered them appropriate for practice. The multidisciplinary discussion form for IPF diagnosis was known by $74 \%$ and considered appropriate by $94 \%$. Diagnosis and management resulted from multidisciplinary discussion in $50 \%$ of the cases. About $58 \%$ of patients were diagnosed with "mild to moderate IPF" as defined by forced vital capacity $\geqslant 50 \%$ of the predicted value and diffusing capacity for carbon monoxide $\geqslant 35 \%$ of predicted. At the time of the survey, $31 \%$ of physicians were using pirfenidone to treat patients with "mild-to-moderately severe IPF" and 30\% generally prescribed no treatment.

Substantial improvement has occurred since the 2011-2012 survey with regard to knowledge of guidelines and proper management of IPF. Early diagnosis still needs to be improved through the network of expert centres.

@ERSpublications

A practice survey reveals important changes in the diagnosis and practical management of IPF http://ow.ly/Tuwr1

This article has supplementary material available from openres.ersjournals.com

Received: June 102015 | Accepted after revision: Oct 122015

Support statement: This study was supported by Fondation Lyon 1 (www.lyonlfondation.org).

Conflict of interest: Disclosures can be found alongside this article at openres.ersjournals.com

The content of this work is (Cthe authors or their employers. Design and branding are @ERS 2015. This article is open access and distributed under the terms of the Creative Commons Attribution Non-Commercial Licence 4.0. 


\section{Introduction}

Idiopathic pulmonary fibrosis (IPF), a rare, irreversible lung disease affecting predominantly males over 60 years of age, leads to progressive dyspnoea and a deterioration of pulmonary function reflected by a progressive decline of forced vital capacity (FVC) and diffusing capacity of the lung for carbon monoxide (DLCO). Progression of the disease results in a serious limitation of physical activities and has a major impact on the quality of life [1]. IPF ultimately leads to death by respiratory failure. The median survival is only 3 years [2].

A first survey of the practical management of IPF, coordinated by the French expert centres (the national reference centre (RC) and the regional competence centres (CCs) for rare lung diseases), was conducted between December 2011 and February 2012 in metropolitan France [3]. About 58\% of the patients followed by 512 pulmonologists who were following at least one patient with IPF had a "mild-to-moderate form of IPF" (as defined by FVC $\geqslant 50 \%$ of predicted value and DLCO $\geqslant 35 \%$ of predicted). Only $35 \%$ of the pulmonologists followed their IPF patients in the context of a coordinated care network (e.g. with established and long-term collaboration between the treating pulmonologist, a pulmonologist from an interstitial lung disease expert centre $(\mathrm{RC} / \mathrm{CC})$, and a radiologist and a pathologist experienced in interstitial lung disease). For the others, access to radiologists and pathologists experienced in this disease was limited. Corticosteroid therapy was the most frequently prescribed treatment.

To meet the needs and expectations expressed by participants in the first survey, a form was developed by the RC/CCs in 2013 to support the conduct of multidisciplinary discussions and to facilitate the diagnosis of IPF (supplementary material) [4]; means to improve the coordination of care pathways and educational programmes at the local, regional and national level were initiated; the number of CCs was increased to better cover most regions in France; and French guidelines for the diagnosis and management of IPF were published by the RC/CCs in December 2013 (also published in English in shorter version) [5]. The French guidelines took into account the marketing authorisation granted to pirfenidone in 2012 for the treatment of mild-to-moderate IPF and updated the literature review as compared to existing international guidelines [6] including for treatments recently demonstrated to be ineffective and/or harmful. These guidelines thus provided French-speaking physicians with a document in their own language, oriented toward practice and decision making

The purpose of the present second survey was to determine whether and how the practice of pulmonologists dealing with IPF patients may have evolved in this context, to describe the existing care network and its possible limitations to access to expert centres or IPF specialists, and to identify possible changes in the expectations and needs of French pulmonologists. The survey also provided an opportunity to gather some data regarding the nature of symptoms leading to IPF suspicion and the time to diagnosis since the onset of symptoms.

\section{Methods}

Organisation of care

The survey was coordinated by the network of French expert centres for rare pulmonary diseases (RC/ CCs), and was conducted by an independent survey company between May 16, 2014 and August 30, 2014. In France, the care of rare pulmonary diseases is coordinated by a RC and 16 CCs throughout France (www.maladies-pulmonaires-rares.fr) that, collectively, are responsible for supporting physicians for the diagnosis of rare pulmonary diseases, including IPF, and for guiding treatment choices. RC/CCs are expert interstitial lung disease centres, and coordinate with respiratory departments from other university hospitals, general hospitals and private practice physicians. Patients with IPF often are seen first by family physicians who refer them to private practice pulmonologists or pulmonologists of general hospitals; patients may then be referred to university hospitals and/or RC/CCs. Multidisciplinary discussions take place in all RC/CCs as well as in some other centres.

\section{Conduct of the survey}

A total of 524 pulmonologists were contacted, including 512 (98\%) who had participated to the first survey. Up to five e-mail reminders or phone recalls were sent to nonrespondents. Finally, 260 of the pulmonologists responded (participation rate, 49.6\%; similar to that of the first survey, 50\%), of whom 223 followed at least one patient with IPF and were invited to answer a questionnaire online or by phone. As the answers of 37 of those pulmonologists were incomplete and not included, data were analysed on the basis of 166 fully completed questionnaires (31.7\% of pulmonologists invited to participate) (figure 1).

\section{Statistics}

Descriptive statistics were performed using Excel, Microsoft Office 2010 (Microsoft Corp., Redmond, WA, USA). The present (second) survey (S2) was not identical to the previous one (S1) and fewer questionnaires were analysed in this survey compared with the previous one (166 in S2 versus 512 in S1). 


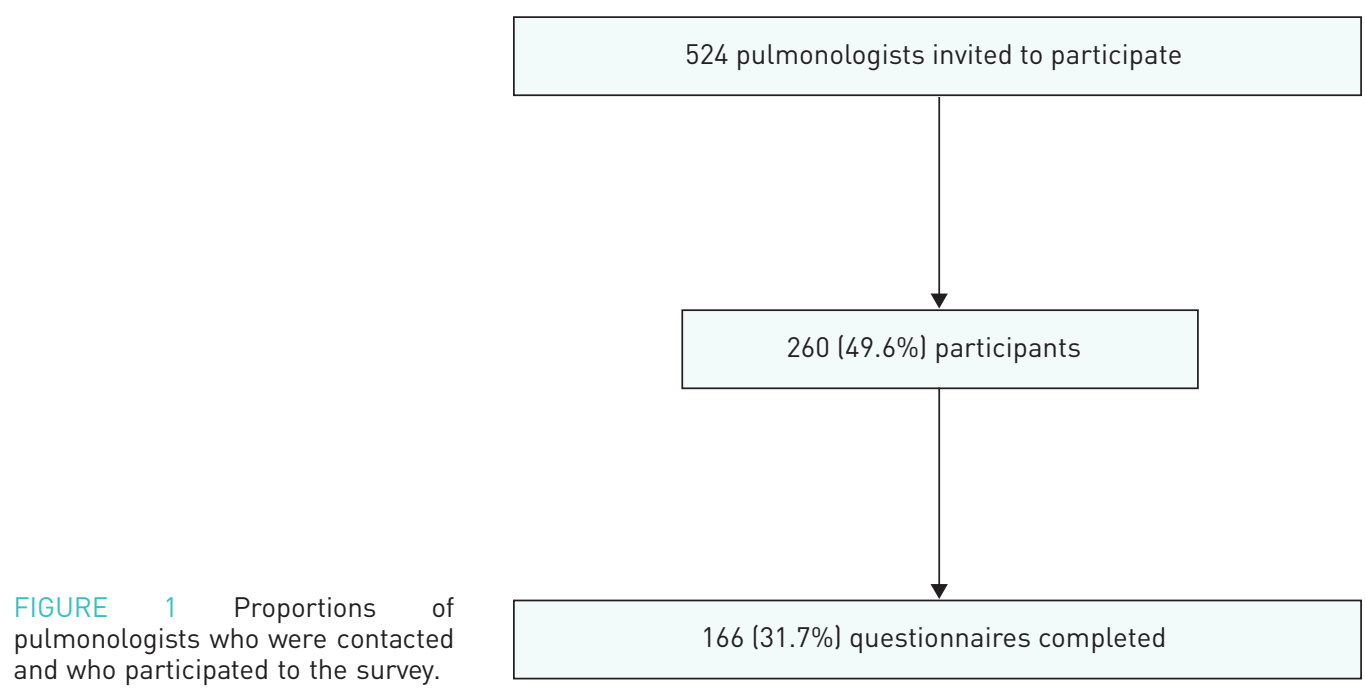

The respondents were not identical. Therefore, no statistical tests were performed for formal comparisons of survey participants between S1 and S2. The distribution of population was compared using the Chi-squared test. A p-value less than 0.05 was considered significant. Suggestions in the survey to improve the diagnosis, management or network of care of IPF patients were phrased by pulmonologists, and verbatim was analysed by topic.

\section{Results}

Survey participants

$72(72 \%)$ of the participants were male. The median age was 52.2 years in males and 45.1 years in females. A majority (65\%) worked in hospitals (12 in the RC or the CCs, 39 in other university hospitals and 57 in general hospitals); 39 (23\%) had a private practice and 19 (11\%) had a mixed type of medical practice (private practice and hospital). Overall, pulmonologists who participated to this survey (S2) were considered representative of physicians managing IPF in France and were generally comparable to those who participated to the first survey (S1) (supplementary table e1).

\section{Patient attendance by care setting}

The mean number of IPF patients currently followed per centre decreased steadily from the RC/CCs ( $\mathrm{n}=53$ ) to the university hospitals $(n=33)$, general hospitals $(n=14)$ and private or mixed practices $(n=9)$, with a mean of 23,16, seven and four new patients seen per year, respectively. Conversely, the percentage of new IPF patients referred directly to the centre was lower in CR/CCs than in other centres $(20 \%$ in the RC/CCs, $27 \%$ in the university hospitals, $62 \%$ in the general hospitals and $100 \%$ by definition in the private or mixed practices), because most patients were initially seen by a private practice pulmonologist or in a general hospital. The proportion of patients with mild-to-moderate IPF was higher in the RC/CCs (63\%) than in the other centres, where it was slightly above $50 \%$ (for differences with results of S1, see table e2).

\section{Knowledge and use of French IPF practical guidelines}

The French practical guidelines for the diagnosis and management of IPF, released in October 2013 [5], adapted and updated from the international guidelines, were known by 133 (92\%) of 144 pulmonologists practicing outside of the RC/CCs and considered suitable by $96 \%$ of them. These guidelines were mostly used for the diagnosis of IPF (44\% of respondents), and less for treatment (25\%), follow-up (24\%), and management of comorbidities and complications (8\%) (table 1).

\section{IPF diagnosis and network of care}

Most (80\%) pulmonologists practicing outside of the RC/CCs indicated that they referred their patients with suspected IPF to one of the expert centres (the national RC in $60 \%$ of cases and one of the 16 CCs in $40 \%$ of cases). Approximately two thirds of the participants (half of those practising outside of the RC/CCs) knew the 2013 multidisciplinary discussion form and used it systematically or often in their practice (table 2).

A high proportion of participants cooperated with other health professionals to establish the diagnosis of IPF (table 3). More than $60 \%$ always or often cooperated with radiologists and pathologists experienced in IPF diagnosis. For $80 \%$ of them, this cooperation always or often occurred in the context of a 
TABLE 1 Proportions of pulmonologists practicing outside of the reference centre/competence centres who used the French recommendations and found them suitable

Pulmonologists \%

$\begin{array}{lc}\text { Use the French recommendations } & \\ \text { Diagnosis } & 44 \\ \text { Treatment } & 25 \\ \text { Follow-up } & 24 \\ \text { Management of comorbidities and complications } & 8 \\ \text { Find the French recommendations suitable } & \\ \text { Highly suitable } & 41 \\ \text { Suitable } & 55 \\ \text { Poorly suitable } & 3 \\ \text { Do not know } & 1 \\ \text { \#:133 answers. } & \end{array}$

multidisciplinary team discussion. Cooperation with radiologists and pathologists experienced in IPF diagnosis markedly increased, from $37 \%$ to $46 \%$ and $34 \%$ to $52 \%$, respectively, between the two surveys.

With the aim of identifying barriers to early diagnosis of IPF, the survey included questions relating to the time frame of symptoms and diagnosis, including the time to diagnosis since the onset of symptoms, the number of physicians consulted before IPF diagnosis and the main clinical or radiological features raising the suspicion of IPF. The time to diagnosis since the onset of symptoms ranged from 9 to 12 months in $35 \%$ of patients and from 18 to 24 months in another 33\%. In 12\%, the delay was more than 24 months. Relatively early diagnosis was rare, within 6 months in $11 \%$ of patients and less than 3 months in $9 \%$ of patients.

Only $3 \%$ of the patients followed by the pulmonologists had not visited any other physician before being diagnosed with IPF. $38 \%$ had already seen their primary care physician and $46 \%$ had already seen two physicians. $11 \%, 1 \%$ and $1 \%$, respectively, had already seen three, four and more than four physicians.

Next, we enquired about the variety of clinical or radiological features that had raised the suspicion of IPF. As expected, almost all (97\%) of the physicians had suspected IPF in patients with exertional dyspnoea; $85 \%$ of physicians had diagnosed IPF in patients with persistent cough, $69 \%$ in patients referred for Velcro-like crackles on pulmonary auscultation and $61 \%$ in patients with typical abnormalities incidentally discovered on high-resolution computed tomography (HRCT) performed for another reason. Finger clubbing, HRCT specifically performed because of IPF suspicion, weight loss and a clinical presentation suggesting IPF exacerbation were mentioned only exceptionally.

\section{Management of mild-to-moderate IPF}

$50 \%$ of pulmonologists practicing outside of the RC/CCs decided the management of their IPF patients in the context of a multidisciplinary team including at least one radiologist and one pathologist (if a

TABLE 2 Proportions of pulmonologists practicing outside of the reference centre/competence centres who used the multidisciplinary discussion form and find it suitable

Pulmonologists \%

$\begin{array}{lr}\text { Use the multidisciplinary discussion form } & \\ \text { Diagnosis } & 44 \\ \text { Treatment } & 25 \\ \text { Follow-up } & 24 \\ \text { Management of comorbidities and complications } & 8 \\ \text { Find the multidisciplinary discussion form suitable } & \\ \text { Highly suitable } & 35 \\ \text { Suitable } & 59 \\ \text { Poorly suitable } & 5 \\ \text { Do not know } & 1\end{array}$

\footnotetext{
\#: 106 answers.
} 
TABLE 3 Proportion of pulmonologists establishing the diagnosis of idiopathic pulmonary fibrosis in cooperation with other health professionals

\begin{tabular}{lcccc} 
Cooperation with & Always & Often & Only when needed & Never \\
\hline Specialised radiologist & 46 & 20 & 19 & 14 \\
Specialised pathologist $^{\#}$ & 52 & 14 & 22 & 12 \\
Multidisciplinary team $^{\text {ๆ }}$ & 60 & 20 & 16 & 4
\end{tabular}

Data are presented as \%. " : pulmonary biopsy for suspected idiopathic pulmonary fibrosis; ${ }^{\text {१ः }}$ comprising at least one pulmonologist, one radiologist and one pathologist.

pulmonary biopsy was performed for suspected IPF), while the RC/CCs and other pulmonologists were involved in $28 \%$ and $14 \%$ of cases, respectively. A very small proportion of pulmonologists decided to manage patients on their own. Compared with S1, these results showed a more frequent use of multidisciplinary teams, while the RC/CCs and other pulmonologists were less frequently involved (Chi-squared 14.89, $\mathrm{p}=0.019$ ) (table 4).

Major changes were seen regarding the pharmacological treatment of mild-to-moderate IPF. Whereas in 2011-2012, the predominant treatment was corticosteroid therapy alone (27\%), or in combination with immunosuppressive therapy or $\mathrm{N}$-acetylcysteine (22\%), the majority of physicians in the second survey prescribed pirfenidone alone $(31 \%)$ or in combination with $N$-acetylcysteine (7\%). Those using corticosteroids in monotherapy or in the context of a triple therapy were no more than $5 \%$ and $2 \%$, respectively. $11 \%$ of the physicians followed patients included in a clinical trial. $30 \%$ generally prescribed no specific treatment for IPF ( $27 \%$ in the previous survey).

\section{Expectations and needs of pulmonologists regarding the diagnosis and management of IPF}

131 (79\%) of the 166 pulmonologists participating in S2 suggested several improvements with regard to the diagnosis, management and follow-up of IPF patients. The main suggestions and wishes expressed by the physicians included a better organisation of cooperation between health professionals (42\%) with tools dedicated to the exchange of information; improvements in professional knowledge, education, and skills (35\%); and a more coordinated research network with access to clinical trials or to centres participating in trials (17\%). In addition, some suggestions were made to improve the multidisciplinary discussion form, including its computerisation, its possible simplification, and/or the addition of practical tools to facilitate the differential diagnosis between IPF and other interstitial lung diseases, especially hypersensitivity pneumonitis, drug-induced interstitial lung disease or occupational lung diseases, and access to a detailed description of typical HRCT abnormalities.

\section{Discussion}

2 years after the first survey, this second survey reveals important changes in the diagnosis and practical management of IPF by French pulmonologists. With a $49.6 \%$ participation rate (and a rate of $31.7 \%$ of invited pulmonologists completing the survey), this survey confirms the interest of French pulmonologists in IPF. The high level of cooperation between pulmonologists and other specialists to confirm the diagnosis of IPF and to design its management, often in the context of a multidisciplinary team discussion, is encouraging. The wide adoption by pulmonologists of a recommended evidence-based treatment for patients with mild-to-moderate IPF must be commended. However, late diagnosis remains the rule and significant progress has to be made in this regard.

TABLE 4 Proportions of pulmonologists ${ }^{\#}$ devising idiopathic pulmonary fibrosis management in cooperation with other idiopathic pulmonary fibrosis specialists

Cooperation with

Specialised centre (RC or CC)

Multidisciplinary team

Other pulmonologists

No cooperation, devising management on their own
Second survey

First survey

Data are presented as \%. ${ }^{*}$ : practicing outside the reference centre (RC)/competence centres (CCs). 
The diagnosis of IPF was frequently established during a multidisciplinary discussion during which the multidisciplinary discussion form made by the RC/CCs was widely used. This was facilitated by the fact that, compared with the first survey, pulmonologists practicing outside of the RC/CCs had easier access to radiologists and pathologists experienced in IPF. The multidisciplinary discussion form (see supplementary material for the French and the English versions) is used by community pulmonologists to collect information on patients who will be referred to an interstitial lung disease centre (RC/CC) with a multidisciplinary discussion team in place and was also provided by RC/CCs as an attempt to facilitate the organisation of new multidisciplinary discussions in hospitals where it was not yet available. However, $40 \%$ of pulmonologists still did not discuss cases in a multidisciplinary discussion, underlying that progress is still needed in the access to experienced specialists. The number of multidisciplinary discussions (present in all $\mathrm{RC} / \mathrm{CCs}$ and in some other centres) has increased in France with the need to better apply diagnostic guidelines and because health authorities required that multidisciplinary discussion takes place for pirfenidone to be prescribed. The organisation of new multidisciplinary discussions in university or general hospitals other than RC/CCs (e.g. outside of expert interstitial lung disease centres) explains that more cases are discussed altogether and that fewer need be referred to RC/CC (table 4). Therefore, multidisciplinary discussions may now involve specialists of different expertise. Interestingly, no guidelines are available internationally or nationally regarding how a multidisciplinary discussion should be organised and what experience is required for coordinating physicians. Whether multidisciplinary discussions always involved at least one experienced pulmonologist, radiologist and pathologist was not explored in the questionnaire. Direct referral of patients with mildly-to-moderately severe IPF to the RC/CCs has decreased, which would confirm their role as tertiary care centres. A better knowledge of the disease and improved skills for differential diagnosis may also contribute to the apparent decrease in the number of diagnosed cases as compared to the first survey (table e1), although no direct comparison should be made.

On a less positive note, the diagnosis was established in more than two thirds of cases between 9 and 24 months after the onset of symptoms, most often after the patients had visited three successive physicians. An earlier diagnosis of IPF has become relevant since controlled clinical trials have demonstrated a reduction in the rate of decline of FVC using pirfenidone [7] or nintedanib [8] (the latter was not available for routine use at the time of the second survey). New data on pirfenidone have been published since the completion of this survey. In a phase 3 randomised controlled trial, pirfenidone significantly reduced the decline in FVC and improved progression-free survival in patients with IPF [9]. In addition, in a pre-specified pooled analysis incorporating results from two previous phase 3 trials, the between-group difference favouring pirfenidone was significant for death from any cause and IPF-related death [9].

Two approaches have been proposed to allow an earlier diagnosis of IPF: investigating any patients with early Velcro crackles at lung auscultation and using current large-scale lung cancer screening strategies with low-dose HRCT for the detection of subclinical interstitial lung disease and especially early IPF [10]. Inspiratory crackles are almost constant in patients with IPF; in most cases, older subjects with Velcro crackles are eventually diagnosed with IPF. Therefore, education of primary care physicians, prompting them to refer their patients with crackles to specialised centres, is warranted. In addition, progress has been made in identifying cases associated with a genetic mutation, especially of the telomerase gene complex, in adults and it is anticipated that an increasing number of family members will be identified, carrying a mutation and potentially presenting a subclinical or early form of IPF [11].

A radical change was seen in the pharmacological treatment of patients with mild-to-moderate IPF. Corticosteroid therapy, which was the mainstay of IPF treatment at the time of the first survey, became very rarely prescribed, while pirfenidone, the first agent with a marketing authorisation for the treatment of mild-to-moderate IPF, became the predominant treatment, prescribed mostly in monotherapy. Percentages in the survey reflect estimates of pulmonologists prescribing a given treatment but cannot be translated into a number of treated or untreated patients. This shift of treatment paradigm was consistent with the therapeutic recommendations of the French practical guidelines for IPF, which took into account the results of major clinical trials published between 2012 and 2014 [12-19], and were made widely available in France in the local language. However, as only $31 \%$ of pulmonologists prescribed pirfenidone, it is possible that treatment guidelines may take longer to be adopted by the community than diagnostic guidelines.

It is clear that IPF management, once intuitive and empirical, has entered an evidence-based era. In this context, regularly updated practical guidelines written in the local language are important to translate the results of clinical research into clinical practice. During the previous survey, the international guidelines (available in English) [6] were known by only $61 \%$ of the pulmonologists, whereas $92 \%$ knew the French practical guidelines during the second survey. Similarly, a number of national guidelines written in local European languages have been published between 2012 and 2013. 
The needs and expectations expressed by the participants of this second survey are in line with those of pulmonologists working in other countries [20] and converge with the patients' wishes [21]. Beyond the advances already achieved, current issues include the possibility to establish an earlier diagnosis, an improvement of the overall management of the disease, a better recognition of symptoms and problems faced by the patients, and better organisation of the care network. The latter depends on an improved collaboration between the primary care physicians, pulmonologists (working in hospital or in private practice), expert centres specialised in rare lung diseases, pharmacists and generally all stakeholders involved in IPF management. All in all, the numerous suggestions collected in this survey identified needs and expectations that can be met with reasonable efforts, thus contributing to further improving the organisation of IPF care.

An obvious limitation of the comparisons made between the two surveys deserves some comments. Fewer items were analysed in this survey compared with the previous one and fewer physicians were surveyed (166 versus 512). Only mean numbers, percentages per centre and trends could be used for comparisons. In addition, a selection bias may have impacted the results of the second survey and the comparisons between the two surveys. Due to this limitation, results of the present survey were presented next to those of the first survey (supplementary material) rather than formally compared, except for questions that were directly identical. Nevertheless, $97 \%$ of the second survey participants had already participated to the previous one; the demographic characteristics of the participants and their distribution among the different types of care centres were similar. The second survey predominantly targeted respondents to the first survey, because we reasoned that nonrespondents would not be involved in the management of patients with IPF or would not respond to the second survey either. Therefore, findings in respondents may be biased toward motivated clinicians and may not be applicable to nonrespondents, a limitation which is shared by all surveys. We nevertheless consider the second survey participants as a representative sample of motivated French pulmonologists attending IPF patients, and the comparisons commented here as a broad picture of changes that occurred between 2012 and 2014. However, whether the changes observed were due to the actions of the expert centres or to an increased interest of pulmonologists in IPF due to the advent of effective treatments cannot be determined. In addition, this survey was not an epidemiological study and does not allow estimation of the number of IPF patients in France. Finally, attempting to generalise our results to other countries or to compare to surveys conducted in other areas $[22,23]$ might be risky because of differences in terms of availability of treatments, educational activities and care organisation.

National and international initiatives have already been planned with the aim of further improving IPF care. A European consensus on IPF diagnosis and treatment is expected very soon and an update of the international therapeutic recommendations for IPF has been published [24]. New initiatives taken in France by the expert centres will include an update of the French practical guidelines for IPF, the production of good practice recommendations and protocols, educational initiatives, the further development of active research programmes, an increased visibility of expert centres for patients and healthcare workers through a website (www.maladies-pulmonaires-rares.fr), and the reinforcement of links between expert centres and patients' associations and European programmes.

\section{References}

1 Tomioka H, Imanaka K, Hashimoto K, et al. Health-related quality of life in patients with idiopathic pulmonary fibrosis - cross-sectional and longitudinal study. Intern Med 2007; 46: 1533-1542.

2 Mura M, Porretta MA, Bargagli E, et al. Predicting survival in newly diagnosed idiopathic pulmonary fibrosis: a 3-year prospective study. Eur Respir J 2012; 40: 101-109.

3 Cottin V, Cadranel J, Crestani B, et al. Management of idiopathic pulmonary fibrosis in France: a survey of 1244 pulmonologists. Respir Med 2014; 108: 195-202.

4 Centre de Référence des Maladies Pulmonaires Rares, Centres de Compétences des Maladies Pulmonaires Rares. Discussion MultiDisciplinaire dans le cadre du diagnostic de la Fibrose Pulmonaire Idiopathique (FPI) [Multidisciplinary discussion within the framework of the diagnosis of idiopathic pulmonary fibrosis (IPF)]. http://splf.fr/wp-content/uploads/2014/08/206015-DIAGNOSTIC_FPI_CR-CC.pdf

5 Cottin V, Crestani B, Valeyre D, et al. Recommandations pratiques pour le diagnostic et la prise en charge de la fibrose pulmonaire idiopathique. Élaborées par le Centre national de référence et les centres de compétence pour les maladies pulmonaires rares sous l'égide de la Société de pneumologie de langue française. [French practical guidelines for the diagnosis and management of idiopathic pulmonary fibrosis. From the National Reference and the Competence centers for rare diseases and the Société de Pneumologie de Langue Française.] Rev Mal Respir 2013; 30: 879-902.

6 Raghu G, Collard HR, Egan JJ, et al. An official ATS/ERS/JRS/ALAT statement: idiopathic pulmonary fibrosis: evidence-based guidelines for diagnosis and management. Am J Respir Crit Care Med 2011; 183: 788-824.

7 Noble PW, Albera C, Bradford WZ, et al. Pirfenidone in patients with idiopathic pulmonary fibrosis (CAPACITY): two randomised trials. Lancet 2011; 377: 1760-1769.

8 Richeldi L, du Bois RM, Raghu G, et al. Efficacy and safety of nintedanib in idiopathic pulmonary fibrosis. $N$ Engl J Med 2014; 370: 2071-2082.

9 King TE Jr, Bradford WZ, Castro-Bernardini S, et al. A phase 3 trial of pirfenidone in patients with idiopathic pulmonary fibrosis. N Engl J Med 2014; 370: 2083-2092. 
10 Cordier JF, Cottin V. Neglected evidence in idiopathic pulmonary fibrosis: from history to earlier diagnosis Eur Respir J 2013; 42: 916-923.

11 Doyle TJ, Hunninghake GM, Rosas IO. Subclinical interstitial lung disease: why you should care. Am J Respir Crit Care Med 2012; 185: 1147-1153.

12 Douglas WW, Ryu JH, Schroeder DR. Idiopathic pulmonary fibrosis: impact of oxygen and colchicine, prednisone, or no therapy on survival. Am J Respir Crit Care Med 2000; 161: 1172-1178.

13 Nagai S, Kitaichi M, Hamada K, et al. Hospital-based historical cohort study of 234 histologically proven Japanese patients with IPF. Sarcoidosis Vasc Diffuse Lung Dis 1999; 16: 209-214.

14 Gay SE, Kazerooni EA, Toews GB, et al. Idiopathic pulmonary fibrosis: predicting response to therapy and survival. Am J Respir Crit Care Med 1998; 157: 1063-1072.

15 Winterbauer RH, Hammar SP, Hallman KO, et al. Diffuse interstitial pneumonitis. Clinicopathologic correlations in 20 patients treated with prednisone/azathioprine. Am J Med 1978; 65: 661-672.

16 Raghu G, Depaso WJ, Cain K, et al. Azathioprine combined with prednisone in the treatment of idiopathic pulmonary fibrosis: a prospective double-blind, randomized, placebo- controlled clinical trial. Am Rev Respir Dis 1991; 144: 291-296.

17 Johnson MA, Kwan S, Snell NJ, et al. Randomised controlled trial comparing prednisolone alone with cyclophosphamide and low dose prednisolone in combination in cryptogenic fibrosing alveolitis. Thorax 1989; 44: 280-288.

18 Collard HR, Ryu JH, Douglas WW, et al. Combined corticosteroid and cyclophosphamide therapy does not alter survival in idiopathic pulmonary fibrosis. Chest 2004; 125: 2169-2174.

19 Pereira CA, Malheiros T, Coletta EM, et al. Survival in idiopathic pulmonary fibrosis - cytotoxic agents compared to corticosteroids. Respir Med 2006; 100: 340-347.

20 Cottin V. Current approaches to the diagnosis and treatment of idiopathic pulmonary fibrosis in Europe: the AIR survey. Eur Respir Rev 2014; 23: 225-230.

21 Wuyts WA, Peccatori FA, Russell AM. Patient-centred management in idiopathic pulmonary fibrosis: similar themes in three communication models. Eur Respir Rev 2014; 23: 231-238.

22 Troy LK, Chapman SA, Lake F, et al. Current Australasian practice for diagnosis and management of idiopathic pulmonary fibrosis: Where are we now? Respirology 2015; 20: 647-653.

23 Collard HR, Loyd JE, King TE Jr, et al. Current diagnosis and management of idiopathic pulmonary fibrosis: A survey of academic physicians. Respir Med 2007; 101: 2011-2016.

24 Raghu G, Rochwerg B, Zhang Y, et al. An Official ATS/ERS/JRS/ALAT Clinical Practice Guideline: Treatment of Idiopathic Pulmonary Fibrosis. An Update of the 2011 Clinical Practice Guideline. Am J Respir Crit Care Med 2015; 192: e3-19. 